\title{
Can Respiratory Therapists Impact COPD Readmissions and Costs?
}

The Patient Protection and Affordable Care Act $^{1}$ has had the most significant impact on the financial operations and viability of hospitals and health systems since the introduction of the Medicare and Medicaid Entitlement Program in the 1960s. For hospitals and health systems, the Patient Protection and Affordable Care Act addresses a fundamental shift from payment for services provided within the acute phase of care to being held accountable across the care continuum. With this expanded focus and systemwide accountability, respiratory therapists (RTs) must transition their focus to align with the new reimbursement system. RTs must move from the traditional in-patient focus to a more holistic one that demonstrates the value of the RT to manage the quality, cost, and patient satisfaction in ways never expected in the traditional acute care sickness model.

Of the changes enacted within the Patient Protection and Affordable Care Act, the Value Based Incentive Program has been shown to have the greatest impact on hospital operations and finances. The 3 programs that comprise this program are the Value Based Purchasing, Hospital Acquired Conditions, and Hospital Readmissions Reduction Programs..$^{2-4}$ The Hospital Readmissions Reduction Program has garnered the attention of every hospital and health system, particularly with regard to the annual penalty increase that now is up to $3 \%$ for hospitals with excessive unplanned readmissions. For the first 3 y of the Hospital Readmissions Reduction Program, the penalties for excessive readmissions increased from 1 to $3 \%$ for the diagnoses of congestive heart failure, pneumonia, and acute myocardial infarction. With the addition of COPD in the fourth year, the program was expanded to include allcause and all-condition unplanned readmissions. ${ }^{5}$ The program explanation and reporting of excessive readmissions are published each year with listings of each hospital and its performance. Hospitals with excessive readmissions, defined as a ratio $>1.0$, receive financial penalties from Medicare based upon their degree of excessive readmissions. ${ }^{6,7}$

Mr Kauffman has disclosed relationships with Monaghan Medical and
Mallinckrodt.

Correspondence: Garry W Kauffman RRT FAARC MPA FACHE, 7001 West Road, Walnut Cove, NC 27052. E-mail: gwkauffman@ hotmail.com.

DOI: $10.4187 /$ respcare.04999
The historical emphasis for short-term acute care hospitals has focused on providing care for in-patients and less so outside the traditional 4 walls of the hospital. With

See the Original Study on Page 1137

the advent of the Patient Protection and Affordable Care Act Hospital Readmissions Reduction Program, this focus has necessarily been broadened to include all post-acute care venues, including emergency services, skilled nursing facilities, urgent care, retail outpatient clinics, assistedliving facilities, home care, hospice care, and others. The challenge for the acute care hospital has been and continues to be managing care across the continuum and seeking to understand the factors that drive care at each level. Specific to readmissions and stay, hospitals and health systems have been attempting to understand causative factors that lead to readmissions. Since the introduction of the diagnosis-related group (DRG) for reimbursement in the 1980s, hospitals and health systems have been making changes in care delivery for the patient as a means of achieving high quality care in concert with care delivery costs that allowed the acute care hospital to survive.

Given that patients with COPD have some of the highest comorbidities (eg, congestive heart failure, acute renal disease, pneumonia), identification of patients with COPD is most challenging. With the addition of COPD to the Hospital Readmissions Reduction Program, the Patient Protection and Affordable Care Act also changed the formula for readmission to include all causes and all conditions. Thus, identifying the patient with COPD is essential to financial operations, with the acknowledgment that allcause and all-condition extends beyond readmissions with the same DRG to include other comorbidities that drive readmissions.

Several previous investigations have evaluated the costeffectiveness of respiratory care protocols and devices. Each of these, involving both COPD patients and other diagnoses, revealed opportunities for reducing operational costs and demonstrating the value of RTs. ${ }^{8-11}$ The study by LaRoché et al ${ }^{12}$ adds to this body of research by examining the potential value of electronic medical record identification of COPD patients. In this study, the authors created a process within their electronic medical record to identify each subject's acuity by implementing a patient-focused 
RT protocol that captured clinical information. They created a clinical scoring tool that was incorporated within a patient assessment and protocol program, with the goal of improving quality of care for COPD patients and assessing the ability to decrease operational costs. The tool incorporated measurement of bedside pulmonary function (ie, $\mathrm{FEV}_{1}$ ) and patient assessment with utilization of a treatment algorithm that directed care to one of 2 treatment regimens. Of note was that the historical process for delivering bronchodilator therapy was predicated on a physician-directed order and nurse-delivered care. The algorithm developed stratified the care delivery based upon the new process, with the direction of care for those of lower acuity receiving bronchodilator therapy delivered by nursing, and those patients with higher acuity receiving bronchodilator therapy delivered by RTs.

The hypothesis of LaRoché et al ${ }^{12}$ was that utilization of an electronic medical record-based screening tool to identify COPD patients and the subsequent new care delivery process would result in a reduction in length of stay, readmission, rapid response deployment, and concomitant cost (or cost avoidance). They report a statistically significant decrease in 30-d readmissions and a decrease in rapid response deployment for those subjects with a primary diagnosis of COPD. Length of stay change was not significant, but a downward trend was noted. This investigation provides both a confirmation of the value of protocols and patient assessment tools with regard to 30 -d readmissions and rapid response deployment as well as provides an insight into potential for length of stay decrease.

There are several limitations as described by the authors, including a small sample size, short time frame, potential variation between primary and secondary diagnoses, lack of validation of the scoring tool and patient-focused respiratory care protocols, and the research design was neither randomized nor blinded. In addition to these limitations as noted by the authors, there are other issues that were not addressed in this investigation and/or that offer opportunity for future investigation. As the authors posit, it is important to understand differences between primary and secondary diagnoses, since the DRG system was based upon resource consumption. ${ }^{13}$ In addition to this, I suggest expansion of COPD to include the specific base DRG and examination of outcomes according to whether the patient was assigned to DRG 190, 191, or 192. This delineation could be done by electronic medical record review and could provide an opportunity to analyze outcomes by base DRG before proceeding with the study according to original design. By utilizing base DRGs, along with true cost data abstraction from the hospital's cost accounting system, we would have more confidence in the impact of such a novel approach to impacting in-patient costs, especially given the paucity of studies involving specific interventions by RTs with regard to quality and cost.
As clinicians know, the diagnosis of COPD can only be determined by the performance of a pulmonary function test. As for the comment on dissection into base DRG, this could be investigated via the electronic medical record to determine patients who had a pulmonary function test documenting air flow obstruction and utilizing this analysis before proceeding with future studies.

Of particular concern, and in response to the authors' acknowledgment that the screening tool was not validated, was the assignment of subjects to treatment regiments by different clinicians (ie, nurses, RTs) who utilized different nebulizers. Future research should include standardization of process and bronchodilator delivery devices between treatment groups. This could be accomplished by selecting the same nebulizer; standardizing the treatment regimen (eg, treatment time, stop at sputter); and ensuring that the number of treatments, patient assessments, and process outcomes (eg, missed treatments, patient refusals) are similar. Although this appears onerous, it is critical in the study design to understand differences in patient response and outcomes. To avoid this complexity, I suggest selection of one nebulizer, a standardized procedure, and delivery by nursing or RT rather than both groups. Finally, although the process for patient assessment and calling for rapid response may exist in this hospital as a standardized process, it is left for readers to make this assumption. I would suggest including indications for which rapid response is called to ensure that there is no process variation between patient groups and/or that any variation is noted. Due to these limitations, it is unclear how the findings of this single-center study will generalize to other settings.

In summary, this investigation provides a novel approach to understanding, identifying, and managing the care of primary and secondary COPD patients. Significant differences were noted as a result of this investigation, and directional trends were noted that should be included in an enhanced research design in the future. I encourage the authors to address their design limitations and those I have mentioned to study the impact of integrating the electronic medical record with a novel patient pulmonary acuity scoring tool and protocols to make a positive impact on readmissions, stay, rapid response deployment, and cost. Readers are encouraged to use the results of the LaRoché study ${ }^{12}$ to position RTs as value-added in the current health-care system.

\section{Garry W Kauffman RRT FAARC MPA FACHE} Kauffman Consulting, LLC Walnut Cove, North Carolina

\section{REFERENCES}

1. The Patient Protection and Affordable Care Act. H.R. 3590. 111th Congress. http://www.gpo.gov/fdsys/pkg/PLAW-111publ148/pdf/ PLAW-111publ148.pdf. Accessed May 16, 2016. 


\section{EDITORIALS}

2. Medicare. Hospital Compare: hospital value-based purchasing. https://www.medicare.gov/hospitalcompare/data/hospital-vbp.html. Accessed May 16, 2016.

3. Medicare.gov. Hospital Compare: hospital-acquired condition reduction program. https://www.medicare.gov/hospitalcompare/ HAC-reduction-program.html. Accessed May 16, 2016.

4. Centers for Medicare and Medicaid Services. Readmissions reduction program. https://www.cms.gov/medicare/medicare-fee-for-servicepayment/acuteinpatientpps/readmissions-reduction-program.html. Accessed May 16, 2016.

5. Centers for Medicare and Medicaid Services. CMS to improve quality of care during hospital inpatient stays. https://www.cms.gov/ Newsroom/MediaReleaseDatabase/Fact-sheets/2014-Fact-sheetsitems/2014-08-04-2.html. Accessed May 16, 2016.

6. Medicare. Hospital Readmissions Reduction Program. https://www. medicare.gov/hospitalcompare/readmission-reduction-program.html. Accessed May 16, 2016.

7. Kaiser Health News (October 2, 2014). Medicare fines 2,610 hospitals in third round of readmission penalties. http://khn.org/news/ medicare-readmissions-penalties-2015/. Accessed May 16, 2016.

8. Emberger JS, Brown JM, Killian L, Maheshwari V. Impact of a breath actuated nebulizer performance improvement on hospital length of stay (abstract). Respir Care 2009;54(11): 1571.

9. Saunders DN. Transitioning to a breath-actuated pneumatic nebulizer in the emergency department and in-patient settings: experience gained from stakeholders involved with the process (abstract). Respir Care 2015;60(10):OF9.

10. Werre ND, Boucher EL, Beachey WD. Comparison of therapistdirected and physician-directed respiratory care in COPD subjects with acute pneumonia. Respir Care 2015;60(2):151-154.

11. Wilson J. Reducing total costs of aerosolized medication delivery using the Aeroeclipse II breath actuated nebulizer (abstract). Respir Care 2011;56(10):1634.

12. LaRoché K, Carl R. Hinkson CR, Thomazin BA, Minton-Foltz P, Carlbom DJ. Impact of an electronic medical record screening tool and therapist-driven protocol on length of stay and hospital readmission for COPD. Respir Care 2016;61(9):1137-1143.

13. Office of Inspector General/Office of Evaluation and Inspections. Medicare hospital prospective payment system: how DRG rates are calculated and updated, August 2001, OEI-09-00-00200. http:// oig.hhs.gov/oei/reports/oei-09-00-00200.pdf. Accessed May 16, 2016. 